\title{
Clinical Medicine 2020 - putting policy into practice
}

The distinct place of a general medical journal such as Clinical Medicine is that we can include an article like the review by Hydes et al looking at the relationship between alcohol consumption and risk of cancer. ${ }^{1}$ It is a continuing curiosity that there exist different attitudes towards the regulation of the tobacco and alcohol industries. This may reflect the differences in the lay perception of the cancer risks caused by smoking compared with alcohol. The authors have therefore posited a question to help quantify the carcinogenic risk of alcohol: how many cigarettes are there in a bottle of wine? Do read the article for the answer.

In a similar vein we are delighted to publish a manuscript like that of Colver et al. ${ }^{2}$ The subject is an emerging one in clinical practice that of the transition of patients from paediatric to adult care (which can continue until the age of 24). Transition has been regarded as the responsibility of children's services but is increasingly a topic of relevance for funders of adult services. The evidence base for implementing transition is poor, and it is this deficit that Colver and colleagues from north-east England have addressed. They have undertaken a body of 10 studies, the Transition research programme, which have been peer reviewed and presented in recent years. This manuscript integrates these interlinked studies to provide an overall summary with implications for funders and providers, as well of course for clinicians. It is a particular strength of the paper that it is presented from a generic service perspective rather than focusing on one technical aspect, and the implications are generalisable to systems throughout the world.

The international dimension of what we publish is reflected in the manuscript from Lund by Spångfors et al looking at NEWS for the detection of in-hospital cardiac arrest. ${ }^{3}$ We receive a large number of manuscripts and correspondence about NEWS, so publications that make it in to Clinical Medicine are of high quality and illustrate a particular clinical point. Using an innovative approach, the Swedish authors look at different timespans in the hours preceding an in-hospital cardiac arrest and also evaluate, using clinical risk classification, the discriminative ability of NEWS among general ward patients. Their findings reflect a dynamic process of deterioration for in-hospital cardiac arrests, providing potential opportunities to intervene. This paper is complemented by one by Nakitende et al. ${ }^{4}$
They report the utility of a range of early warning scores studied in prospective fashion in an acute setting in a single hospital in Uganda. Comparing simpler and more complex scores (requiring more equipment and interpretation) the findings may be a surprise to you. Clinical Medicine is getting fuller, with more original research, alongside our regular CME section (cardiovascular medicine) and, in this edition, the Harveian oration by the geneticist Sir John Burn entitled 'Prediction and prevention in the genomic era'. In addition, we are delighted to feature more case material, abidingly popular (as reflected by the featured quote from a reader on the front cover). This case material is in the form of images, lessons of the month and acute medical cases. The new decade will see the content of the journal develop further. We are delighted to have appointed two outstanding trainee physicians with a talent for writing. They will contribute a new regular feature, 'Research in brief' - concise reviews of key papers in major specialist and general medical journals. Involvement of trainees in the publishing aspect of the Royal College of Physicians' activity is something we are keen to grow, so please do keep an eye out for the opportunities as they emerge on the website and social media.

\section{References}

1 Hydes T], Williams R, Sheron N. Exploring the gap in the public's understanding of the links between alcohol and cancer. Clin Med 2020;20:4-7.

2 Colver A, Rapley T, Parr JR et al. Facilitating transition of young people with long-term health conditions from children's to adults' healthcare services - implications of a 5 -year research programme. Clin Med 2020;20:74-80.

3 Spångfors M, Molt M, Samuelson K. In-hospital cardiac arrest and preceding National Early Warning Score (NEWS): A retrospective case-control study. Clin Med 2020;20:55-60.

4 Nakitende I, Nabiryo J, Namujwiga T, Wasingya-Kasereka L, Kellett J. Do different patient populations need different early warning scores? The performance of nine different early warning scores used on acutely ill patients admitted to a low-resource hospital in sub-Saharan Africa. Clin Med 2020;20:67-73.

Anton Emmanuel Editor-in-chief

$\begin{array}{ll}\text { Members of the editorial board } \\ \text { Michael Almond } & \text { Anton Emmanuel } \\ \text { Cono Ariti } & \text { Kate Evans } \\ \text { Paul Belcher } & \text { Johanna Feary } \\ \text { Tessa Cacciottolo } & \text { Maggie Hammersley } \\ \text { Rodger Charlton } & \text { Dylan Harris } \\ \text { Tim Chevassut } & \text { Tevfik Ismail } \\ \text { Tahseen Chowdhury } & \text { Vikas Kapil } \\ \text { Elaine Dennison } & \text { Neelam Kumar } \\ \text { Albert Edwards } & \text { Alexandra Lake }\end{array}$

Yash Mahida Nick Manning-Cork Chris Marguerie Martin McKee Andrew Medford Rahul Mukherjee Mehool Patel Rajan Pooni Gerrard Phillips
Roby Rakhit Sushma Saksena Avan Sayer Philip Smith Angela Star Cameron Swift Rhys Thomas 